\title{
Modeling of the Stepped Leader Initiation Process in an Altitude Triggered Lightning
}

\author{
Bo Zhang, Bin Chen, Lihua Shi, and Qiang Chen \\ National Key Laboratory on Electromagnetic Environmental Effects and Electro-Optical Engineering, \\ PLA University of Science and Technology, Nanjing 210007, China
}

Correspondence should be addressed to Bo Zhang; emczhangbo@163.com

Received 30 September 2015; Accepted 10 April 2016

Academic Editor: Anna Vila

Copyright (c) 2016 Bo Zhang et al. This is an open access article distributed under the Creative Commons Attribution License, which permits unrestricted use, distribution, and reproduction in any medium, provided the original work is properly cited.

In an altitude triggered lightning, the potential and charge distribution of the triggering wire, a floating conductor, is unknown and changeable during the triggering process, which makes it difficult to simulate an altitude triggered lightning in a numerical work. To solve this problem, a 3D altitude triggered lightning model is developed in this paper, which contains two parts, a Thundercloud Model which is time-dependent and nonhydrostatic and takes 27 kinds of microphysical processes into consideration and a Triggering Model in which a charge conservation equation is introduced to describe the floating conductor and to ensure the overall neutrality of the leader channel. Numerical results of the stepped leader initiation process are given, which are in good agreement with experiment observations.

\section{Introduction}

Many rocket triggered lightning experiments have been done to study the propagation mechanism of the stepped leader [16]. These triggered lightnings can be classified into two kinds, namely, the classical triggered lightning and the altitude triggered lightning $[7,8]$. The main difference between these two methods is the way the triggering wire, a conductor, is connected to the ground. In a classical triggered lightning, the triggering wire is connected to the ground directly. But in an altitude triggered lightning, the triggering wire is connected to the ground through a dielectric wire, which makes it difficult to simulate an altitude triggered lightning in a numerical work because the electric potential and the induced charge distribution along the triggering wire are unknown and changeable for a simulator.

Recently, much research progress has been made on the modeling of the classical triggered lightning. Dong and Ge developed a two-dimensional plane symmetric model for the simulation of classical triggered lightning [9]. Baba and Rakov developed their classical triggered lightning model based on the finite difference time domain (FDTD) method to examine the current, charge transfer, and the fieldreduction effect during the lightning process [10]. However, numerical research on the altitude triggered lightning is still rare. Lalande et al. proposed an altitude triggered lightning model to study the lightning connection process to a ground structure [11]. In their model, the background electric field produced by the thundercloud was assumed to be vertical and deduced from measurement. The field and potential distribution distorted by the presence of the floating wire were calculated using the Charge Simulation Method (CSM); that is, the charge density along the triggering wire was fixed to a certain value empirically.

In this paper, a numerical model is developed to study the altitude triggered lightning physically. This model is composed of two main parts, a Thundercloud Model and a Triggering Model. The Thundercloud Model is used to produce a background electric field. In the past, the thundercloud charge distribution was often simplified as a tripole model [12-14] in which the thundercloud was assumed to be three axisymmetric cylinders with different polarity and the microphysical process of the cloud particles was not considered. Currently a 2D Cartesian cumulus model [15], developed by the Chinese Academy of Meteorological Sciences, is used to study the relationship between lightning types and the distribution of space charge and electric potential [16]. In this paper, a 3D dynamics and electrification coupled model [17] is 


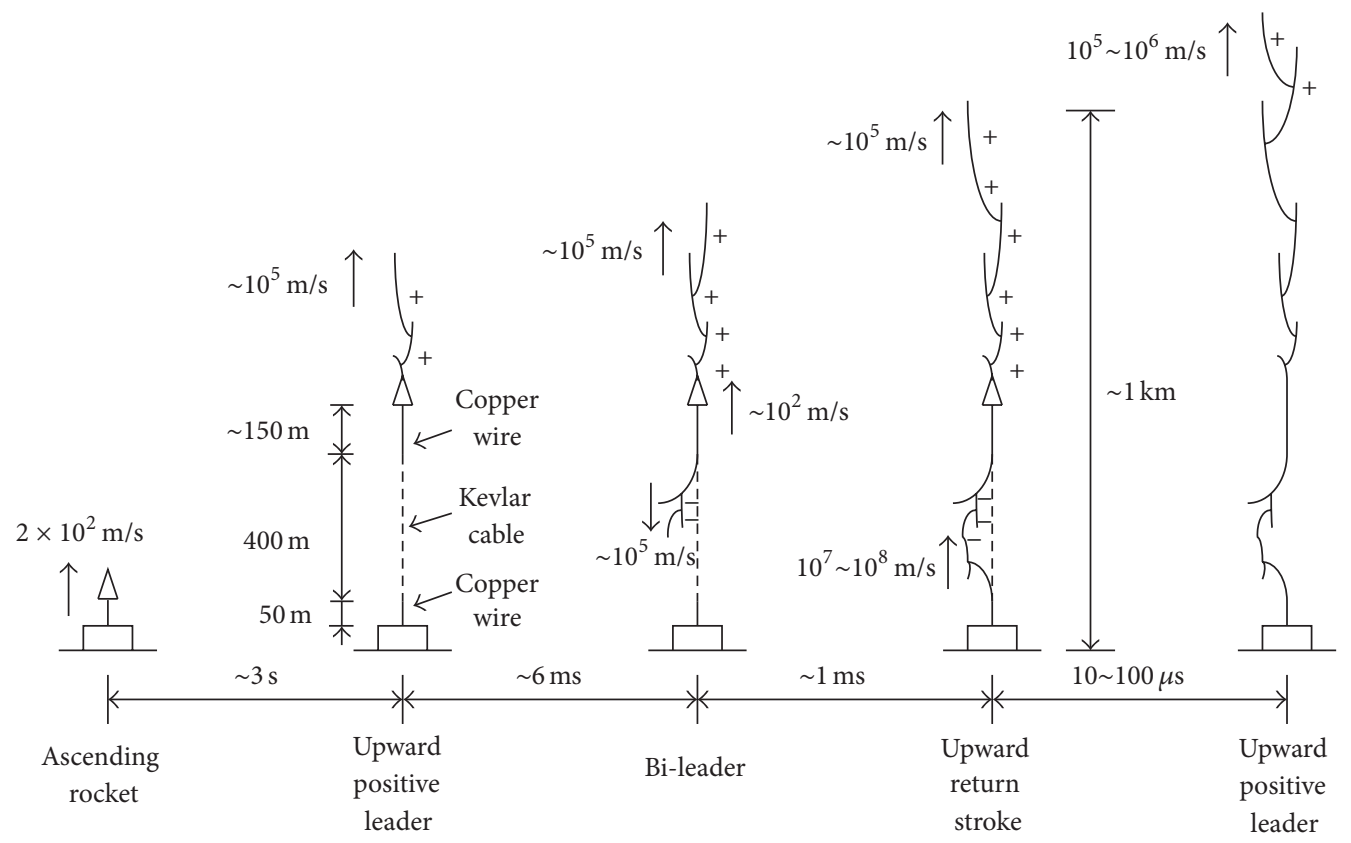

FIGURE 1: Sequence of events involved in the formation of the first stroke in altitude triggered lightning.

employed, which is time-dependent and nonhydrostatic and takes 27 kinds of microphysical processes into consideration. In the Triggering Model, the Dielectric Breakdown Model (DBM) [18-20], which is a stochastic fractal model, is used to simulate the propagation of the stepped leader. To solve the problem of floating conductor, a charge conservation equation is introduced to describe the triggering wire. Also, this equation can be used to ensure the overall neutrality of the leader channel. To validate the proposed model, numerical results of an altitude triggered lightning in the initial process are given, which are in good agreement with the observations in $[6,11]$.

\section{Modeling}

The triggering process of an altitude triggered lightning is shown in Figure 1 according to [11]. With the launch of a small rocket, the lower grounded copper wire is pulled out from the spool with a length of $50 \mathrm{~m}$, followed by a $200 \sim 400 \mathrm{~m}$ Kevlar and an upper floating copper wire. When the upper copper wire has been trailed out over a sufficient length, an upward positive leader is initiated from the tip of the rocket and propagates toward the cloud. A few milliseconds later, a downward negative stepped leader is developed from the bottom of the triggering wire and propagates toward the ground $[6,11]$. The lower copper wire is used to direct the connection of the downward negative leader to an equipped grounded structure, realized by an upward connecting positive leader initiated from the tip of the lower copper wire. When the junction process completes, an upward positive return stroke will occur and clear most of the induced charge in the leader channel. As a result, the potential of the whole channel is changed. The return stroke process is finished in a very short time, and then the upward positive stepped leader continues propagating toward the cloud with a higher speed.

2.1. The Thundercloud Model. To simulate an altitude triggered lightning, a background thundercloud is needed firstly. In this paper, the $3 \mathrm{D}$ dynamics and electrification coupled model in [17] is employed to simulate the thundercloud. The model is based on [15], the inductive charged process is adopted from [21], and the noninductive charged process is adopted from [22]. Finally, the following time-dependent, nonhydrostatic equation set for the model is derived in fully compressible form:

$$
\frac{\partial u_{i}}{\partial t}+u_{j} \frac{\partial u_{i}}{\partial x_{j}}=-c_{p} \bar{\theta}_{v} \frac{\partial \pi^{\prime}}{\partial x_{j}}+\delta_{i 3} g A+\frac{\rho_{T}}{\rho_{a}} E_{i}+D_{u_{i}},
$$

where $A=\left[\theta_{v}^{\prime} / \bar{\theta}_{v}+0.61 q_{v}^{\prime}-q_{t}\right]$,

$$
\begin{aligned}
& \frac{\partial \theta}{\partial t}+u_{j} \frac{\partial \theta}{\partial x_{j}}=S_{\theta}+D_{\theta}, \\
& \frac{\partial \pi^{\prime}}{\partial t}+u_{j} \frac{\partial \pi^{\prime}}{\partial x_{j}}=-\frac{\overline{c^{2}}}{c_{p} \bar{\rho} \overline{\theta_{0}^{2}}} \frac{\partial \bar{\rho} \bar{\theta}_{u_{j}}}{\partial x_{j}}-\frac{R_{d}}{c_{v}} \pi^{\prime} \frac{\partial u_{j}}{\partial x_{j}} \\
&+\frac{c^{2}}{c_{p} \theta_{v}^{2}} \frac{d \theta_{v}}{d t}+D_{\pi^{\prime}}, \\
& \frac{\partial q_{v}}{\partial t}+u_{j} \frac{\partial q_{v}}{\partial x_{j}}= S_{q_{v}}+D_{q_{v}}, \\
& \frac{\partial M_{x}}{\partial t}+u_{j} \frac{\partial M_{x}}{\partial x_{j}}= \frac{1}{\bar{\rho}} \frac{\partial \bar{\rho} V_{x} M_{x}}{\partial z}+S_{M_{x}}+D_{M_{x}},
\end{aligned}
$$




$$
\begin{aligned}
\frac{\partial n_{ \pm}}{\partial t}+u_{j} \frac{\partial n_{ \pm}}{\partial x_{j}}= & \mp \frac{\partial\left(\mu_{ \pm} E_{x} n_{ \pm}\right)}{\partial x} \mp \frac{\partial\left(\mu_{ \pm} E_{y} n_{ \pm}\right)}{\partial y} \\
& \mp \frac{\partial\left(\mu_{ \pm} E_{z} n_{ \pm}\right)}{\partial z}+S_{n_{ \pm}}+D_{n_{ \pm}} \\
\frac{\partial q_{x e}}{\partial t}+u_{j} \frac{\partial q_{x e}}{\partial x_{j}}= & \frac{1}{\bar{\rho}} \frac{\partial \bar{\rho} V_{x} q_{x e}}{\partial z}+S_{x e}+D_{x e} \\
\rho_{T} & =e\left(n_{+}-n_{-}\right)+\sum q_{x e} \\
\frac{\partial^{2} \varphi}{\partial^{2} x}+\frac{\partial^{2} \varphi}{\partial^{2} y}+\frac{\partial^{2} \varphi}{\partial^{2} z} & =-\frac{\rho_{T}}{\varepsilon} \\
E_{x}= & -\frac{\partial \varphi}{\partial x} \\
E_{y} & =-\frac{\partial \varphi}{\partial y} \\
E_{z} & =-\frac{\partial \varphi}{\partial z}
\end{aligned}
$$

where $M_{x}=\left[\begin{array}{ll}q_{x} & N_{x}\end{array}\right]$ and the subscripts $x=[c, r, i, s, g, h]$ denote the cloud water, rain water, ice crystal, snow, graupel, and hail, respectively. $D$ and $S$ denote the turbulent mixing and the source term in subgrid scale, respectively. $q_{x e}$ denotes the charge density of the precipitation particles $\left(\mathrm{C} / \mathrm{m}^{3}\right) . \varepsilon$ is the electric permittivity of the air. $e$ is the electron charge. $\varphi$ denotes the electric potential field. $E_{x}, E_{y}$, and $E_{z}$ are the electric fields $(\mathrm{V} / \mathrm{m})$ in different directions, respectively. $\rho_{T}$ is the total charge density $\left(\mathrm{C} / \mathrm{m}^{3}\right)$ of the space. $n_{ \pm}$denotes the density of positive and negative free ions. The other physical quantities are the same as [15].

2.2. The Triggering Model. Since the stepped leader channel is far away from the outer boundary of the computational space in the initial process, as shown in Figure 2, the potential of the outer boundary can be considered as constant. However, the inner boundary, namely, the triggering wire and the leader channel, is changeable during the process. It is difficult to tell the potential and charge distribution along the inner boundary. To solve this problem, a charge conservation equation is introduced to describe the inner boundary.

Assuming that the potential of the triggering wire is $\varphi_{0}$, we can calculate the potential of the stepped leader channel as follows:

$$
\varphi_{m}=\varphi_{0} \pm \sum_{i=1}^{m} E_{\text {in }} d_{i}
$$

where $m$ is the number of the step, $d_{i}$ is the length of step $i$, and $E_{\text {in }}$ is the inner electric field of the stepped leader.

For the whole computational space, the potential distribution can be decided by Poisson's equation:

$$
\nabla^{2} \varphi=-\frac{\rho_{T}}{\varepsilon} .
$$

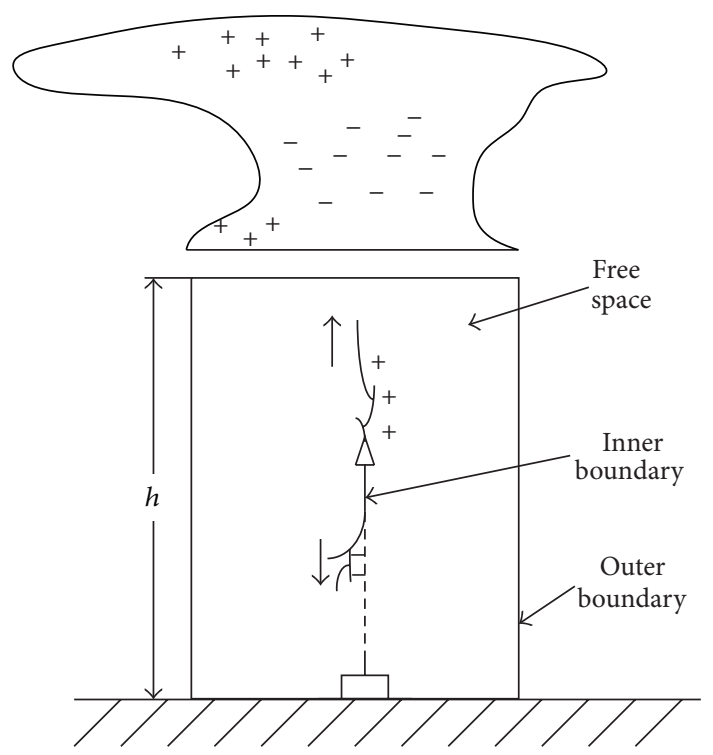

Figure 2: The Triggering Model of the stepped leader in initial process.

And the boundary conditions are

$$
\begin{aligned}
\left.\varphi\right|_{\partial \Omega_{1}} & =\psi, \\
\int_{\Omega_{2}} \rho_{s} d s & =0,
\end{aligned}
$$

where $\Omega_{1}$ is the outer boundary, $\Omega_{2}$ is the inner boundary, and $\rho_{s}$ is the surface charge density of the triggering wire and leader channel.

For free space, $\rho_{T}=0$. Poisson's equation is changed into Laplace's equation $\nabla^{2} \varphi=0$. Using the central difference method, Laplace's equation can be written as

$$
\begin{gathered}
\frac{\left.\varphi\right|_{i+1, j, k}-\left.2 \varphi\right|_{i, j, k}+\left.\varphi\right|_{i-1, j, k}}{\Delta x^{2}} \\
+\frac{\left.\varphi\right|_{i, j+1, k}-\left.2 \varphi\right|_{i, j, k}+\left.\varphi\right|_{i, j-1, k}}{\Delta y^{2}} \\
+\frac{\left.\varphi\right|_{i, j, k+1}-\left.2 \varphi\right|_{i, j, k}+\left.\varphi\right|_{i, j, k-1}}{\Delta z^{2}}=0 .
\end{gathered}
$$

When $\Delta x=\Delta y=\Delta z$, (7) can be rewritten as

$$
\begin{aligned}
& \left.\varphi\right|_{i-1, j, k}+\left.\varphi\right|_{i, j-1, k}+\left.\varphi\right|_{i, j, k-1}-\left.6 \varphi\right|_{i, j, k}+\left.\varphi\right|_{i+1, j, k} \\
& \quad+\left.\varphi\right|_{i, j+1, k}+\left.\varphi\right|_{i, j, k+1}=0 .
\end{aligned}
$$

For the inner boundary, according to the boundary condition between conductor and dielectric, we have

$$
\rho_{s}=\varepsilon E_{\vec{n}}=-\varepsilon \frac{\partial \varphi}{\partial \vec{n}}
$$


where $\vec{n}$ is vertical to the surface of the inner boundary. Substituting (9) into (6), we have

$$
\int_{\Omega_{2}} \frac{\partial \varphi}{\partial \vec{n}} d s=0
$$

Using the direction cosines, (10) can be written as

$$
\begin{aligned}
& \sum_{m=1}^{N}\left[\frac{\left.\varphi\right|_{i \pm 1, j, k} ^{m}-\left.\varphi\right|_{i, j, k} ^{m}}{\Delta x}|\cos \alpha|\right. \\
& +\frac{\left.\varphi\right|_{i, j \pm 1, k} ^{m}-\left.\varphi\right|_{i, j, k} ^{m}}{\Delta y}|\cos \beta| \\
& +\frac{\left.\left.\varphi\right|_{i, j, k \pm 1} ^{m}-\left.\varphi\right|_{i, j, k} ^{m}|\cos \gamma|\right] \Delta s_{m}=0}{\Delta z}
\end{aligned}
$$

where $N$ is the total step number and $\alpha, \beta$, and $\gamma$ are the direction angles. When $\cos \alpha>0(\cos \beta$ or $\cos \gamma)$, the positive sign is used. Otherwise, the negative sign is used. When $\Delta x=$ $\Delta y=\Delta z$, (11) can be rewritten as

$$
\begin{aligned}
\sum_{m=1}^{N} & {\left[\left(\left.\varphi\right|_{i \pm 1, j, k} ^{m}-\left.\varphi\right|_{i, j, k} ^{m}\right)|\cos \alpha|\right.} \\
& +\left(\left.\varphi\right|_{i, j \pm 1, k} ^{m}-\left.\varphi\right|_{i, j, k} ^{m}\right)|\cos \beta| \\
& \left.+\left(\left.\varphi\right|_{i, j, k \pm 1} ^{m}-\left.\varphi\right|_{i, j, k} ^{m}\right)|\cos \gamma|\right] \Delta s_{m}=0 .
\end{aligned}
$$

The equation systems (8) and (12) can be written as a matrix equation

$$
\mathbf{A x}=\mathbf{b}
$$

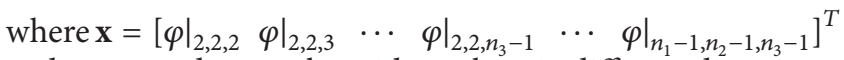
and $n_{1}, n_{2}$, and $n_{3}$ are the grid numbers in different directions.

To solve (13) efficiently, the Successive Overrelaxation method is used. Assume that $\mathbf{A}=\mathbf{P}-\mathbf{N}$, where $\mathbf{P}=\mathbf{D} / \omega+\mathbf{L}$ is a nonsingular matrix, $\mathbf{N}=(1 / \omega-1) \mathbf{D}-\mathbf{U}, \mathbf{L}, \mathbf{D}$, and $\mathbf{U}$ are the lower triangular matrix, diagonal element, and upper triangular matrix of $\mathbf{A}$, respectively, and $\omega$ is the relaxation factor

$$
\omega=\frac{6}{\left(3+\sqrt{9-(\cos (\pi /(l-1))+\cos (\pi /(m-1))+\cos (\pi /(n-1)))^{2}}\right)} .
$$

The iterative equation of (13) can be obtained as

$$
\begin{aligned}
\mathbf{x}^{n+1}= & \mathbf{P}^{-1} \mathbf{N} \mathbf{x}^{n}+\mathbf{P}^{-1} \mathbf{b} \\
= & (\mathbf{D}+\omega \mathbf{L})^{-1}[(1-\omega) \mathbf{D}-\omega \mathbf{U}] \mathbf{x}^{n} \\
& +\omega(\mathbf{D}+\omega \mathbf{L})^{-1} \mathbf{b} .
\end{aligned}
$$

Once the potential field of the whole computational space is solved, the surface charge density of the inner boundary can be solved by $\rho_{s}=-\varepsilon \nabla^{2} \varphi$, and the development of the stepped leader can be decided by the DBM method [20].

\section{Numerical Results and Discussion}

Based on the proposed model, an altitude triggered lightning is simulated. The numerical results contain two parts. Firstly, a thundercloud is calculated according to the electrification scheme of the Thundercloud Model, in which the charge distribution changes over time. The time-dependent potential produced by the thundercloud is used as the background field for the lightning triggering process. Secondly, the lightning triggering process is computed according to the Triggering Model, in which the initial process of the stepped leader is presented. During the initial process, the background electric field is distorted and enhanced locally by the introduction of the triggering wire.

3.1. The Charge Distribution of the Thundercloud. The development of the thundercloud at vertical plane $x=19 \mathrm{~km}$ is shown in Figure 3, where the $y$-axis denotes the horizontal distance, the $z$-axis denotes the altitude of the space, and the $E_{z}$ axis denotes the vertical electric field in the thundercloud. The unit of time is minute. We can see that at $t=20 \mathrm{~min}$, the vertical electric field $E_{z}$ has three centers in different polarities, positive, negative, and positive, respectively, from high altitude to low altitude. The negative center in the middle is much larger than the positive ones. At $t=30 \mathrm{~min}$, the amplitudes of $E_{z}$ get larger, and the centers get closer to the ground, due to the movement of the charged particles which are falling toward the ground with different speed $v_{i}$, where $i$ represents different kinds of particles. At $t=40 \mathrm{~min}$, due to the nonuniformity of collisions and neutralization among different particles, the centers are mixed and broken into several smaller ones. At $t=50 \mathrm{~min}$, the lower positive center is almost eliminated, and the smaller centers develop into three major ones, two upper positive centers and one lower negative center. The extremum of the negative center is $E_{z}=-124.2 \mathrm{kV} / \mathrm{m}$, while the positive extremum is $E_{z}=65.6 \mathrm{kV} / \mathrm{m}$. At this point, a negative cloud-to-ground lightning may probably happen. After $t=50 \mathrm{~min}$, the thundercloud gets weaker and finally comes to the end with the falling of the charged particles to the ground.

Compared with the long-lasting thunderstorm, the altitude triggered lightning is a transient process which is expected to be conducted at some moment before the nature lightning happens. In this example, the triggering process is conducted at $t=25 \mathrm{~min}$. The background fields at this moment are given in Figure 4 (plane $y=18 \mathrm{~km}$ ), including 


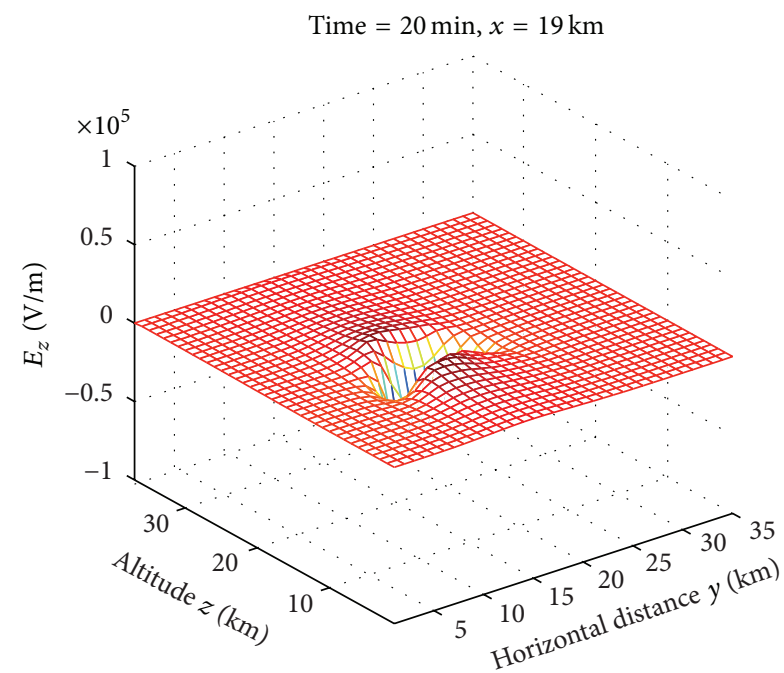

(a)

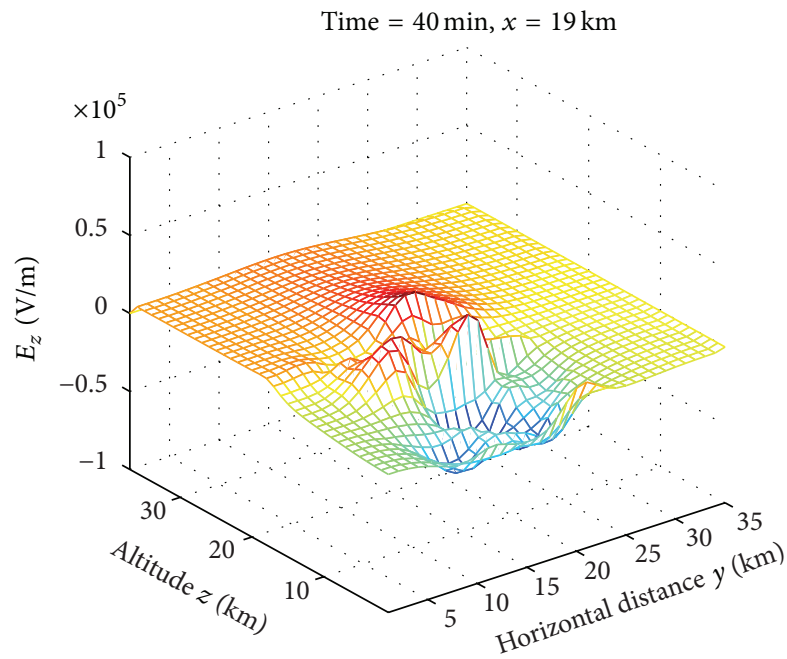

(c)

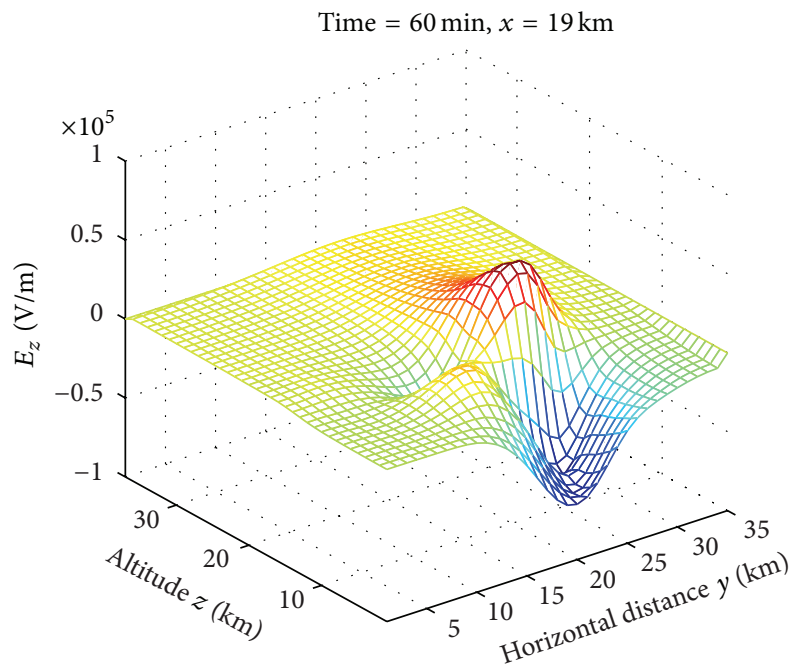

(e)

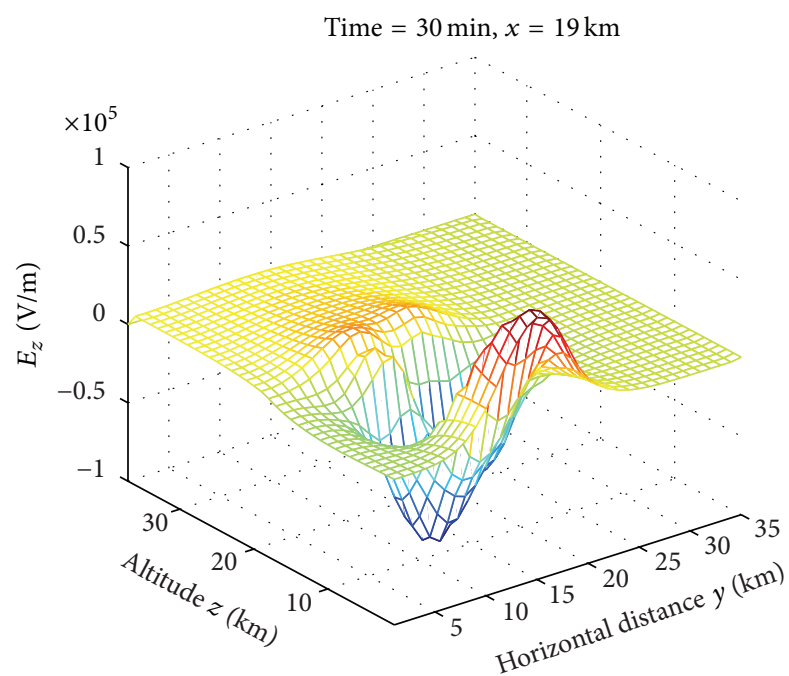

(b)

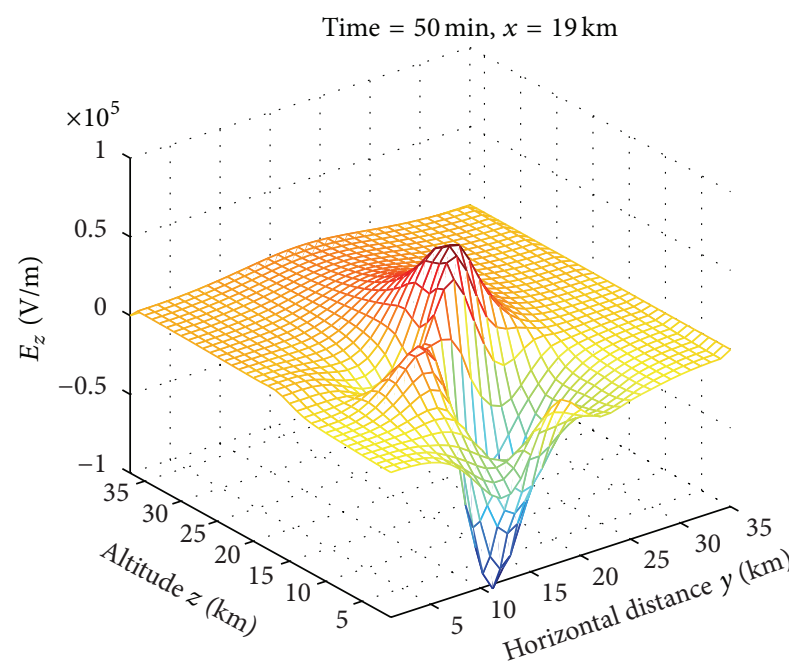

(d)

Time $=70 \mathrm{~min}, x=19 \mathrm{~km}$

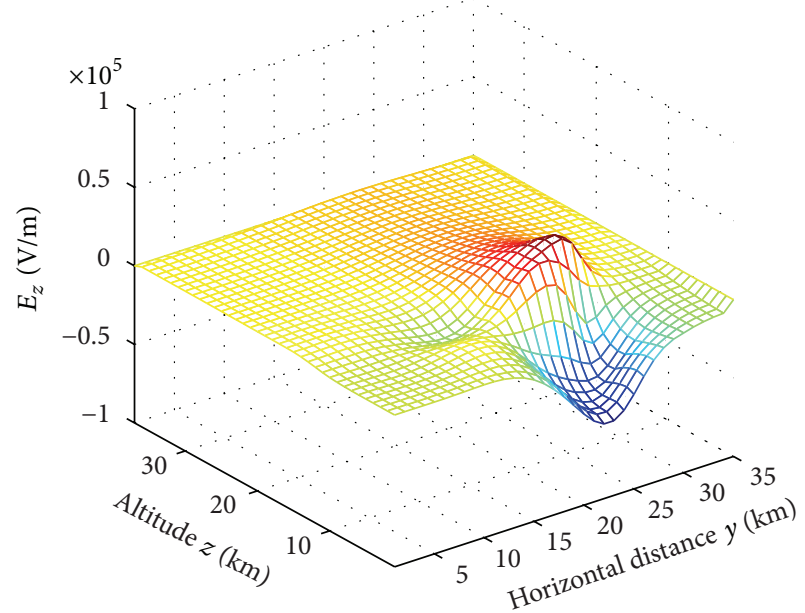

(f)

Figure 3: Profile of the vertical electric field $E_{z}$ in the thundercloud at plane $x=19 \mathrm{~km}$. 


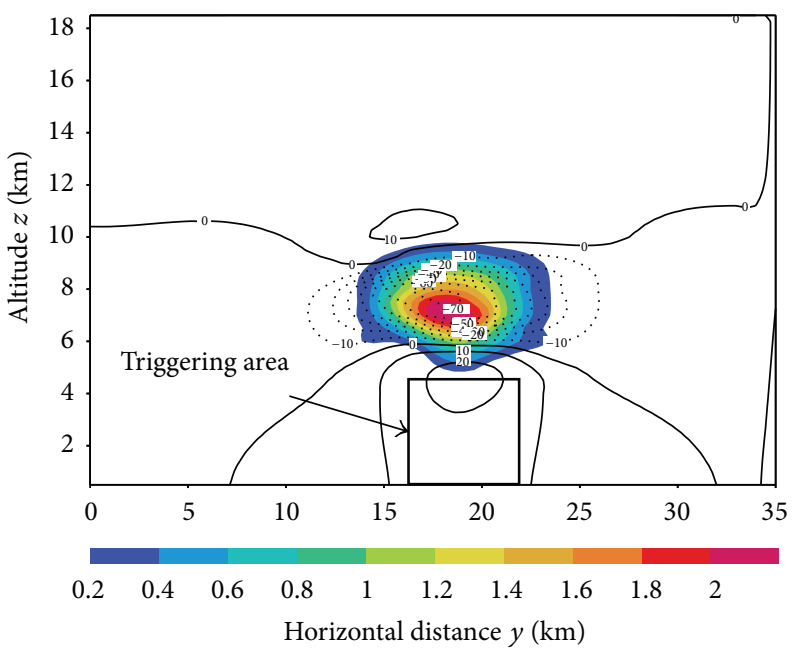

FIgURE 4: Vertical electric field $E_{z}$ and the density of graupel $\rho_{g}$ at plane $y=18 \mathrm{~km}, t=25 \mathrm{~min}\left(E_{z}: \mathrm{km}, \rho_{g}: \mathrm{g} / \mathrm{m}^{3}\right)$.

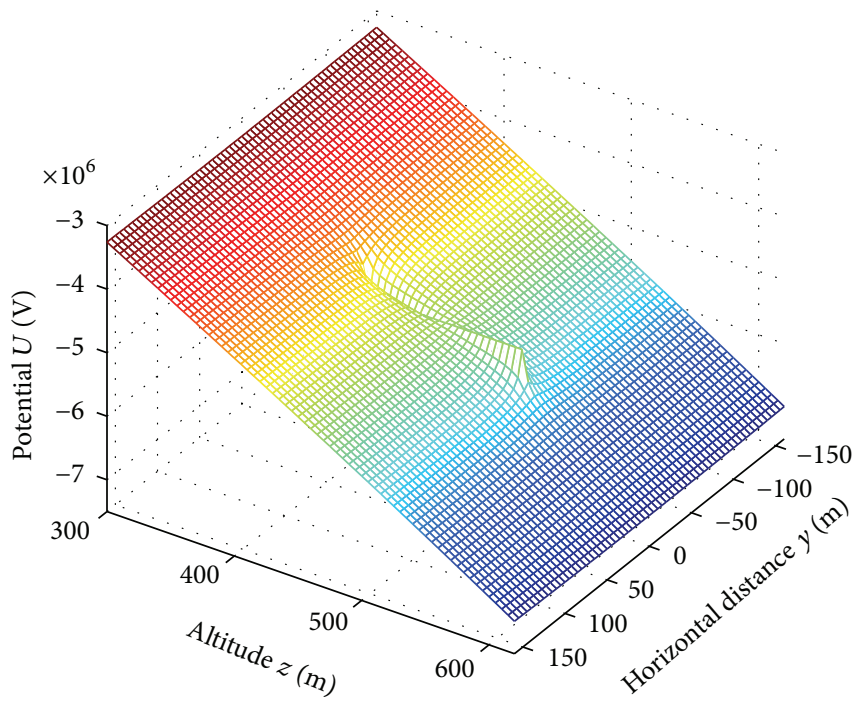

(a)

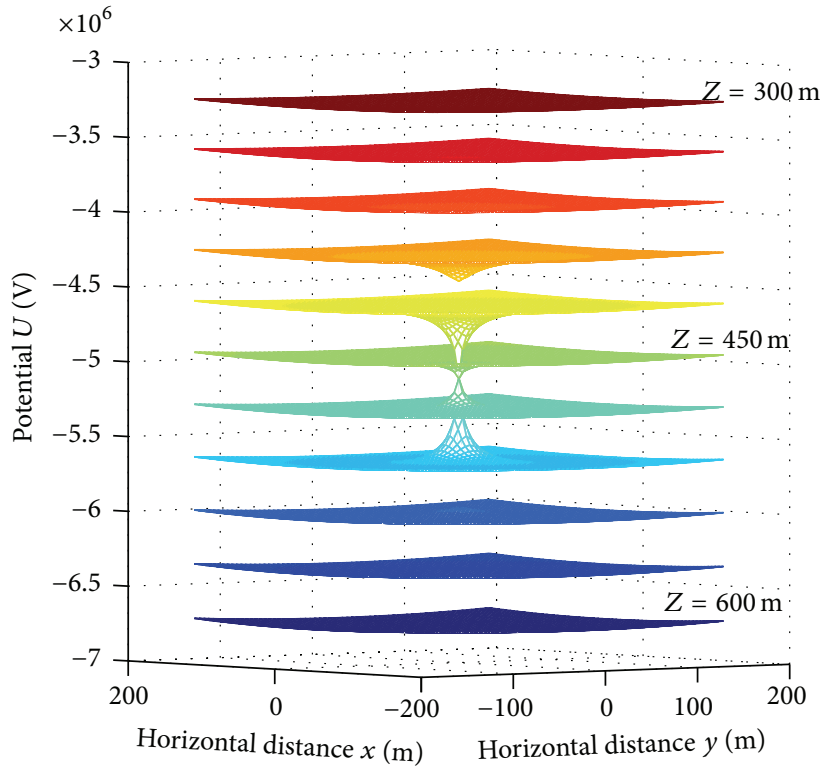

(b)

FIGURE 5: Local potential field near the triggering wire in the view of (a) vertical plane and (b) horizontal plane.

the vertical electric field $E_{z}$ and the density of graupel $\rho_{g}$. The negative and positive extremums of $E_{z}$ are -73.5 and $27.3 \mathrm{kV} / \mathrm{m}$, respectively, and the extremum of $\rho_{g}$ is $2.15 \mathrm{~g} / \mathrm{m}^{3}$. It can be seen that the triggering area contains no charged particles at this moment. Therefore, Poisson's equation $\nabla^{2} \varphi=$ $-\rho / \varepsilon$ for the triggering area can be simplified into Laplace's equation $\nabla^{2} \varphi=0$, except for the triggering wire. It has to be mentioned that the center of the Triggering Model $(0,0,0) \mathrm{km}$ on the ground is set at $(19,18,0) \mathrm{km}$ of the Thundercloud Model.

3.2. Calculation of the Triggering Wire. When the triggering wire is introduced into the space by the rocket, the local electric field near the wire is distorted and enhanced.
According to the proposed Triggering Model, the potential of the triggering wire and the local area near the wire can be calculated. Figure 5 shows the local potential field near the wire at a transient moment during the triggering process. It can be seen that the triggering wire is an equipotential body in the figure with a potential $U=-5.10 \times 10^{6} \mathrm{~V}$, and the electric fields near the ends of the wire are enhanced most. These phenomena are in good agreement with the theoretical analysis.

Since the potential of the local area near the triggering wire is solved, the electric field at the surface of the wire can be solved by $E=-\nabla \varphi$, and the surface charge density of the wire can be calculated by $\rho=-\varepsilon \nabla^{2} \varphi$. Figure 6(a) shows that the potential of the triggering wire decreases nearly in a linear rule with the rise of the rocket (10 $\mathrm{m}$ per time step). 


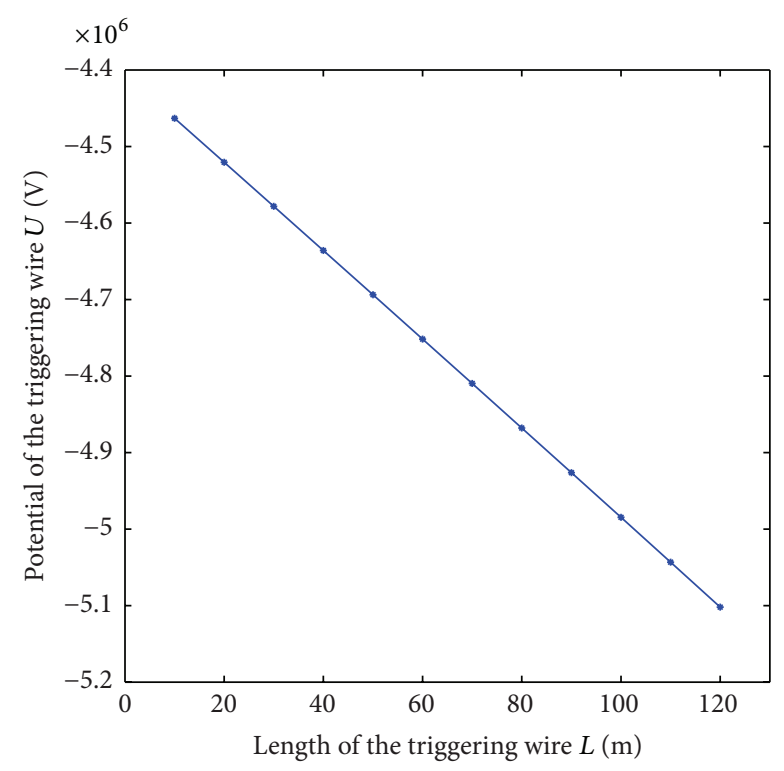

(a)

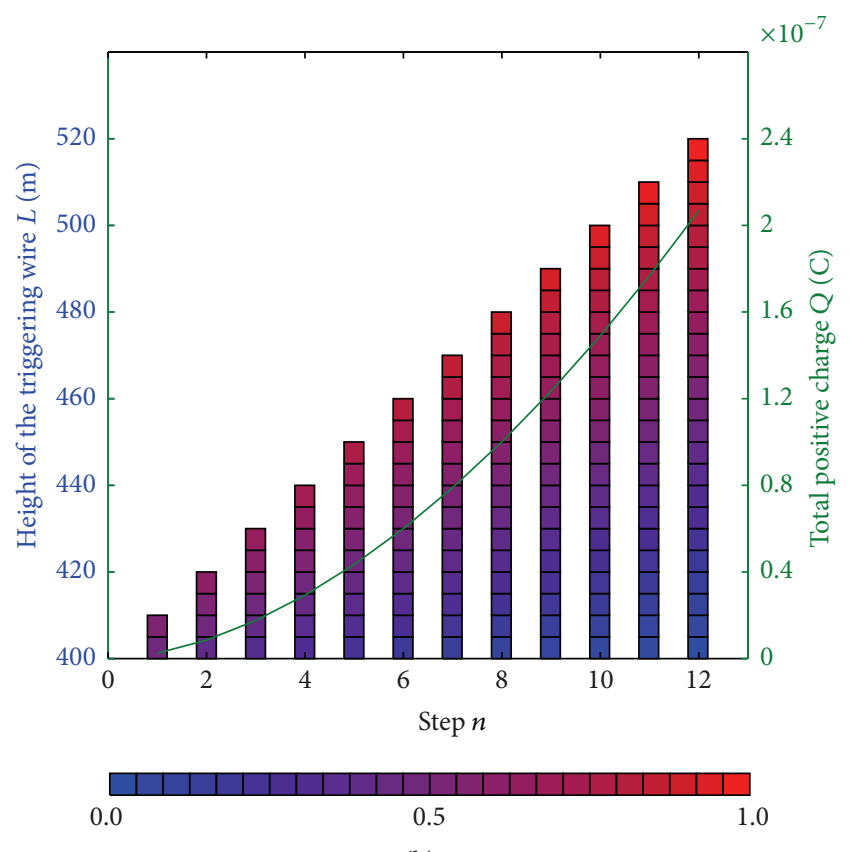

(b)

Figure 6: Variations of the triggering wire during before the first stepped leader, (a) the potential and (b) the induced charge.

It is believed that the change of the potential is affected directly by the background potential field produced by the thundercloud which is almost linear at altitude $200 \sim 800 \mathrm{~m}$. The potential drops from $-4.46 \times 10^{6} \mathrm{~V}$ to $-5.10 \times 10^{6} \mathrm{~V}$ during 12 time steps. At the end of the 12th step, the vertical electric fields $E_{z}$ at the high and low ends of the wire are $89.768 \mathrm{kV} / \mathrm{m}$ and $-88.322 \mathrm{kV} / \mathrm{m}$, respectively. The positive electric field has exceeded the positive threshold value of the stepped leader $\left(E^{+}=80 \mathrm{kV} / \mathrm{m}\right)$ by $10 \%$, and the first leader is initiated from the tip of the rocket, moving toward the cloud. Figure 6(b) shows the variation of the surface charge density

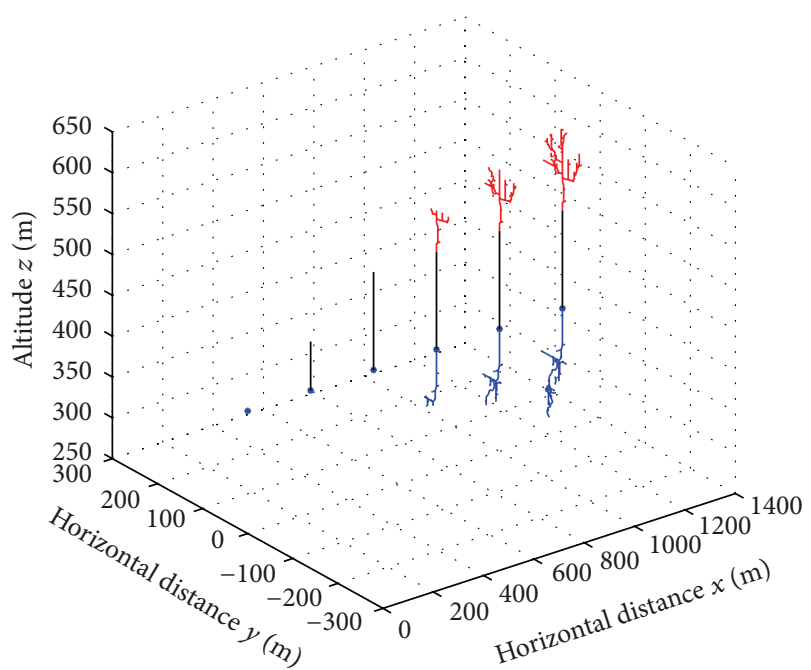

Figure 7: Development of the stepped leader with a lot of bifurcations.

and the total positive charge on the triggering wire. The value of surface charge density is presented by colour in the figure where red (1.0) is equal to $6.21 \times 10^{-7} \mathrm{C} / \mathrm{m}^{2}$ and blue (0.0) is equal to $-6.13 \times 10^{-7} \mathrm{C} / \mathrm{m}^{2}$. The surface charge density grows with the extension of the triggering wire and always has a maximum value on the ends. The curve in the figure denotes the variation of the total positive induced charge on the triggering wire. Different from the potential line, the rule of the total positive induced charge is more like a quadratic curve. The total positive induced charge is $2.067 \times 10^{-7} \mathrm{C}$ before the first stepped leader is initiated.

3.3. Development of the Stepped Leader. Once the stepped leader is initiated, it will develop in a very high speed, much faster than the rocket. As a result, the rocket and the triggering wire can be treated as stationary objects. As shown in Figure 7, the triggering wire stops extending when the stepped leader begins to grow. To make a clear graphic, the triggering system is shifted along the $x$-axis in the order of time. The stepped leader shows abundant bifurcations just like the structure of a nature lightning. The negative leader grows toward the ground with a quite good directivity, while the positive leader is divided into three main branches and each of them may develop into the lightning channel potentially.

To study the initiation process quantitatively, a simulative Lightning Mapping Array (LMA) is used to record the stepped leader, as shown in Figure 8. Figure 8(a) shows the altitude of each stepped leader which is newly added to the leader channel. It is obvious that the initiation of the upward positive leader is earlier than the downward negative leader. By checking the data recorded, we find that the interval between the initiations of the two directional leaders is 22 steps. It is believed that the electric field on the positive end of the triggering wire exceeds the initial threshold $E^{+}$ at first, while the negative electric field on the other end is not enough to initiate a leader. With the development of the positive stepped leader, the electric field on the negative end 


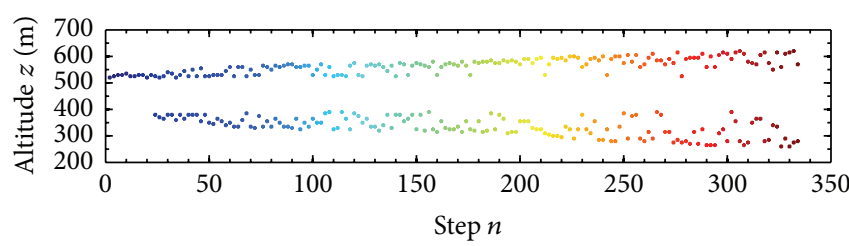

(a)

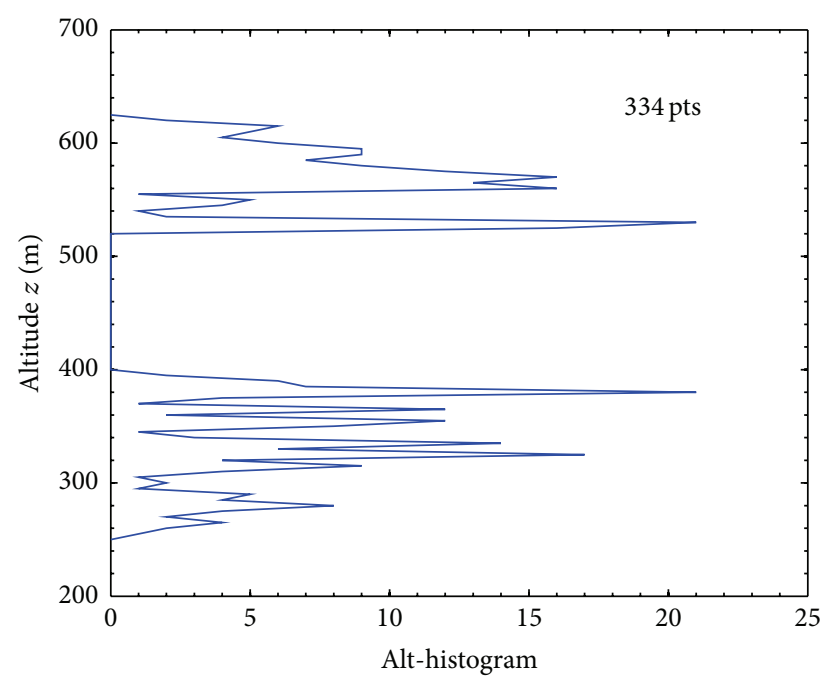

(c)

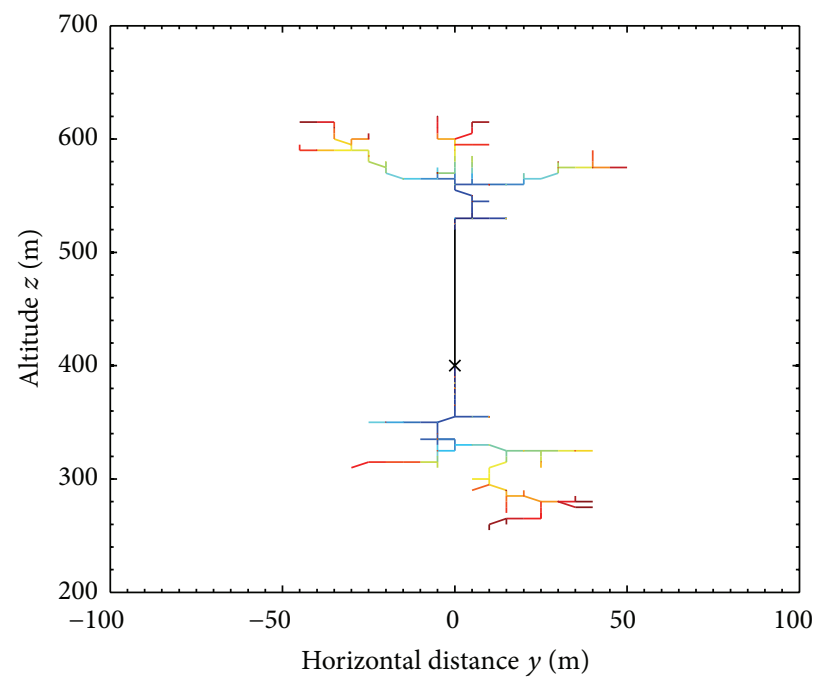

(b)

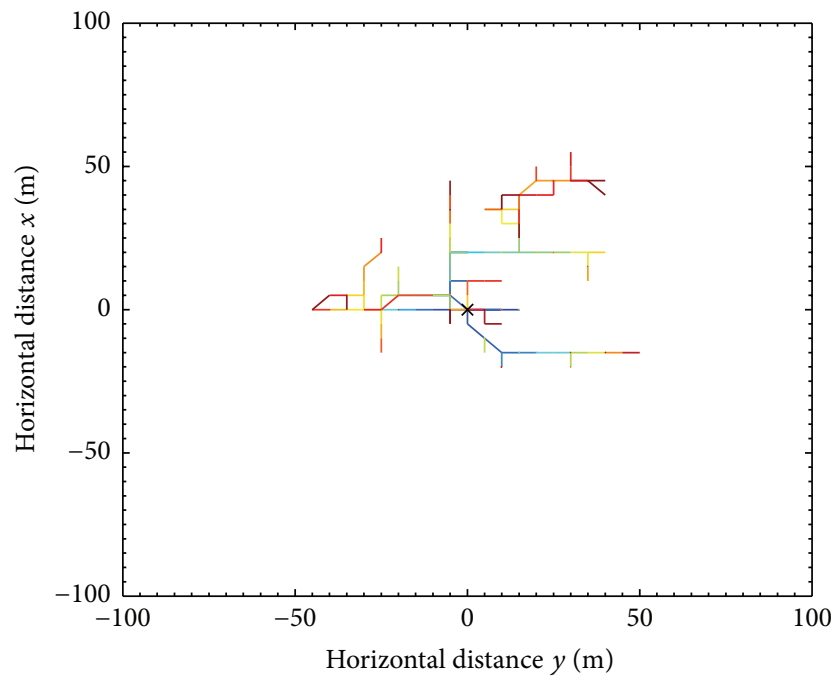

(d)

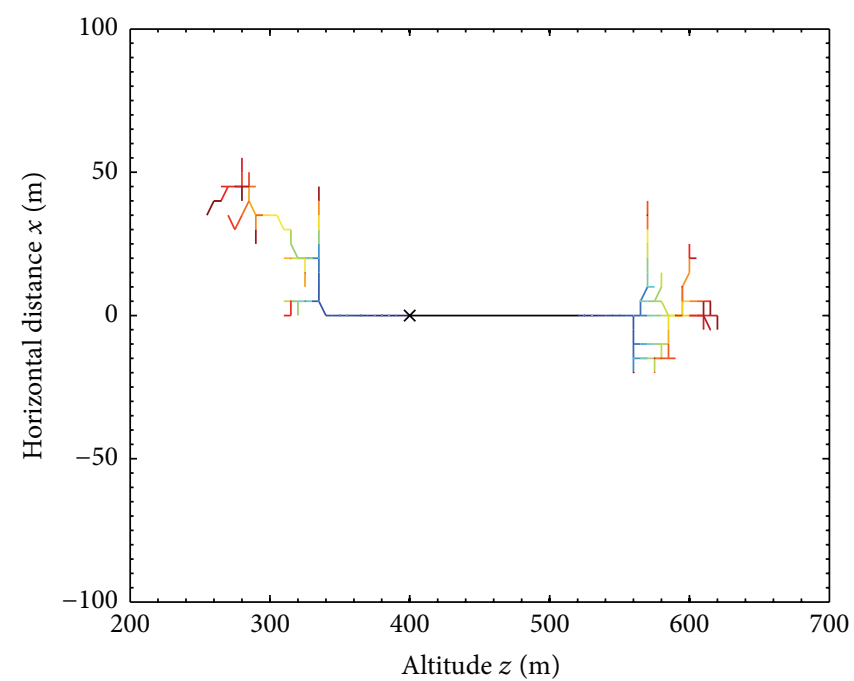

(e)

FIGURE 8: Lightning Mapping Array (LMA) data of the stepped leader at initiation stage. 


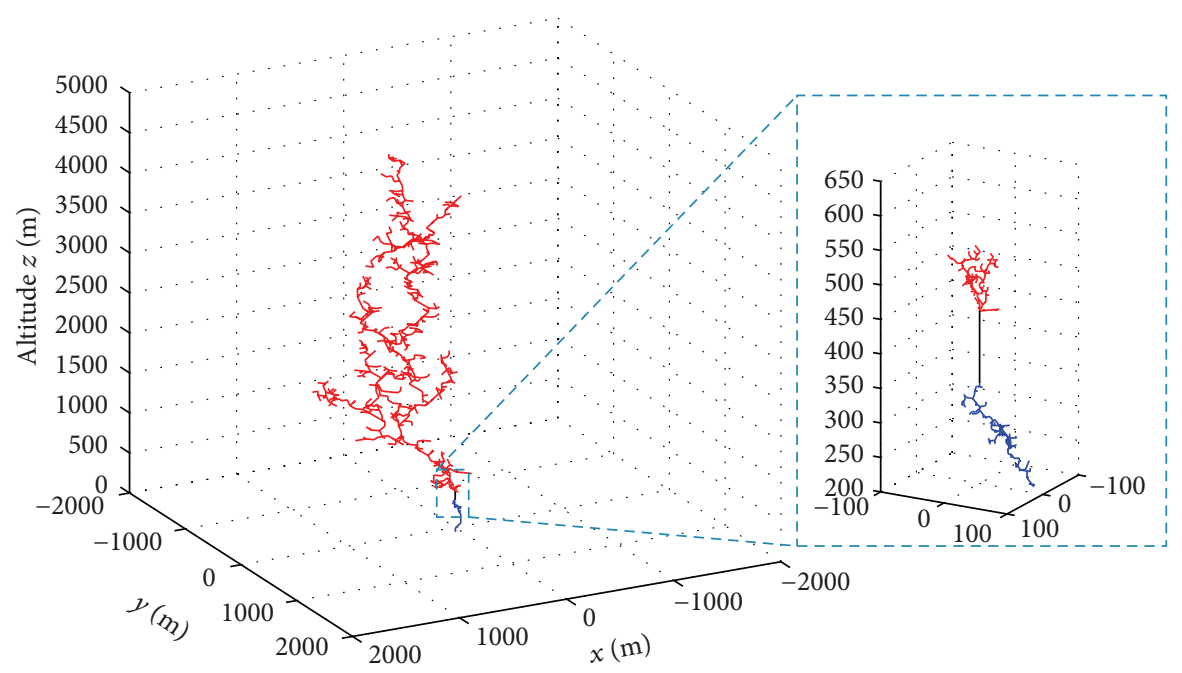

FIgURE 9: Map of the lightning channel and the fine resolution area near the triggering wire.

is enhanced more. At a certain moment, the negative initial threshold $\left(E^{-}=-100 \mathrm{kV} / \mathrm{m}\right)$ is exceeded and a negative stepped leader is initiated from the bottom of the wire. Since the electric fields on the ends of the triggering wire have a similar value at the beginning, the determining factor of the interval in initiation is the difference in leader mechanism [5] which results in different initial thresholds. These phenomena agree well with the observations in $[6,11]$. Figures $8(\mathrm{~b}), 8(\mathrm{c})$, and $8(\mathrm{e})$ show the projections of the leader channel in planes $y-z, y-x$, and $x-z$, respectively, where the scale of the altitude is twice the horizontal ones. And the colour of the channel denoting the sequence of time is in correspondence with Figure 8(a). The newly developed leaders are at the end of the channel. From Figure 8(b), one can see that the positive leader channel breaks into three major braches, and each of them may potentially be the main lightning channel. On the other hand, the negative stepped leader develops more vertically with a clear main channel. Figure $8(d)$ shows the cumulative number of the stepped leader at different altitude. The total number of the stepped leader is 334, among which the number of the positive stepped leader is 178 , distributing from altitude $520 \sim 625 \mathrm{~m}$ with a maximum value 21 at altitude $530 \mathrm{~m}$, and the number of the negative stepped leader is 156 , distributing from altitude $400 \sim 260 \mathrm{~m}$ with a maximum value 21 at altitude $385 \mathrm{~m}$.

Figure 9 shows the main lightning channel triggered by the triggering wire within the computational domain. The channel shows a character of fractal. The resolution for the whole computational domain is $50 \mathrm{~m}$, and the interpolation method is used to change the resolution to $5 \mathrm{~m}$ in the local area near the triggering wire, as shown in the partially enlarged figure.

\section{Conclusions}

To solve the floating conductor problem in the altitude triggered lightning, a 3D numerical model is proposed in this paper. The model is composed of two parts, a Thundercloud Model and a Triggering Model. The Thundercloud Model, which takes 27 kinds of microphysical processes into consideration, is used to produce a background electric field for the Triggering Model. In the Triggering Model, the DBM method is used to simulate the propagation of the stepped leader, and a charge conservation equation is introduced to describe the floating conductor. Numerical results of the proposed model are in good agreement with the observations in real altitude triggered lightnings $[6,11]$.

\section{Competing Interests}

The authors declare that there are no competing interests regarding the publication of this paper.

\section{References}

[1] Z. Saleh, J. Dwyer, J. Howard et al., "Properties of the X-ray emission from rocket-triggered lightning as measured by the Thunderstorm Energetic Radiation Array (TERA)," Journal of Geophysical Research: Atmospheres, vol. 114, no. 17, Article ID D17210, 2009.

[2] J. D. Hill, M. A. Uman, M. Stapleton, D. M. Jordan, A. M. Chebaro, and C. J. Biagi, "Attempts to create ball lightning with triggered lightning," Journal of Atmospheric and SolarTerrestrial Physics, vol. 72, no. 13, pp. 913-925, 2010.

[3] E. Petrache, M. Paolone, F. Rachidi et al., "Lightning-induced currents in buried coaxial cables: a frequency-domain approach and its validation using rocket-triggered lightning," Journal of Electrostatics, vol. 65, no. 5-6, pp. 322-328, 2007.

[4] P. Lalande, A. Bondiou-Clergerie, G. Bacchiega, and I. Gallimberti, "Observations and modeling of lightning leaders," Comptes Rendus Physique, vol. 3, no. 10, pp. 1375-1392, 2002.

[5] R. Jiang, X. Qie, C. Wang, and J. Yang, "Propagating features of upward positive leaders in the initial stage of rocket-triggered lightning," Atmospheric Research, vol. 129-130, pp. 90-96, 2013.

[6] W. T. Lu, Y. J. Zhang, X. J. Zhou et al., "Simultaneous optical and electrical observations on the initial processes of altitudetriggered negative lightning," Atmospheric Research, vol. 91, no. 2-4, pp. 353-359, 2009. 
[7] V. A. Rakov, M. A. Uman, K. J. Rambo et al., "New insights into lightning processes gained from triggered-lightning experiments in Florida and Alabama," Journal of Geophysical Research Atmospheres, vol. 103, no. 12, pp. 14117-14130, 1998.

[8] D. Zheng, Y. J. Zhang, W. T. Lu et al., "Characteristics of return stroke currents of classical and altitude triggered lightning in GCOELD in China," Atmospheric Research, vol. 129-130, pp. 6778, 2013.

[9] W. S. Dong and Z. M. Ge, "The numerical simulation of artificially triggering lightning," Plateau Meteorology, vol. 14, no. 2, pp. 141-150, 1995 (Chinese).

[10] Y. Baba and V. A. Rakov, "Simulation of corona at lightningtriggering wire: current, charge transfer, and the field-reduction effect," Journal of Geophysical Research: Atmospheres, vol. 116, no. 21, Article ID D21115, 2011.

[11] P. Lalande, A. Bondiou-Clergerie, P. Laroche, and G. L. Bacchiega, "Modeling of the lightning connection process to a ground structure," Tech. Rep. ONERA-TAP-96-69, 1996.

[12] V. A. Rakov and M. A. Uman, Lightning: Physics and Effects, Cambridge University Press, New York, NY, USA, 2003.

[13] P. Krehbiel, W. Rison, R. Thomas et al., "Thunderstorm charge studies using a simple cylindrical charge model, electric field measurements, and lightning mapping observations," Eos, Transactions American Geophysical Union, vol. 85, no. 47, abstract AE23A-0843, 2004.

[14] J. A. Riousset, V. P. Pasko, P. R. Krehbiel, R. J. Thomas, and W. Rison, "Three-dimensional fractal modeling of intracloud lightning discharge in a New Mexico thunderstorm and comparison with lightning mapping observations," Journal of Geophysical Research: Atmospheres, vol. 112, no. 15, Article ID D15203, 2007.

[15] Z. J. Hu and G. F. He, "Numerical simulation of microphysical processes in cumulonimbus part I: microphysical model," Acta Meteorologica Sinica, vol. 2, no. 4, pp. 471-489, 1988.

[16] Y. Tan, S. Tao, Z. Liang, and B. Zhu, "Numerical study on relationship between lightning types and distribution of space charge and electric potential," Journal of Geophysical Research Atmospheres, vol. 119, no. 2, pp. 1003-1014, 2014.

[17] A. P. Sun, M. H. Yan, Y. J. Zhang, and H. F. Zhang, "Numerical study of thunderstorm electrification with a 3-D dynamics and electrification coupled model I: model description and parameterization of electrical processes," Acta Metallurgica Sinica, vol. 60, no. 6, pp. 722-731, 2002 (Chinese).

[18] L. Niemeyer, L. Pietronero, and H. J. Wiesmann, "Fractal dimension of dielectric breakdown," Physical Review Letters, vol. 52, no. 12, pp. 1033-1036, 1984.

[19] L. Niemeyer, L. Ullrich, and N. Wiegart, "The mechanism of leader breakdown in electronegative gases," IEEE Transactions on Electrical Insulation, vol. 24, no. 2, pp. 309-324, 1989.

[20] N. Femia, L. Niemeyer, and V. Tucci, "Fractal characteristics of electrical discharges: experiments and simulation," Journal of Physics D: Applied Physics, vol. 26, no. 4, pp. 619-627, 1993.

[21] C. Chiu, "Numerical study of cloud electrification in an axisymmetric, time-dependent cloud model," Journal of Geophysical Research, vol. 83, no. 10, pp. 5025-5049, 1978.

[22] T. Takahashi, "Riming electrification as a charge generation mechanism in thunderstorms," Journal of the Atmospheric Sciences, vol. 35, no. 8, pp. 1536-1548, 1978. 


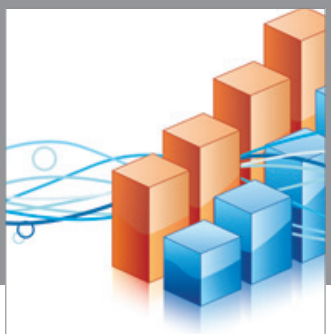

Advances in

Operations Research

vatem alat4

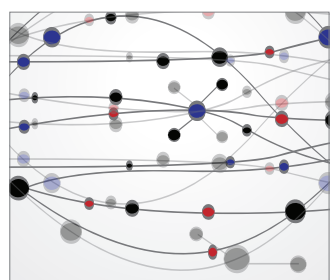

\section{The Scientific} World Journal
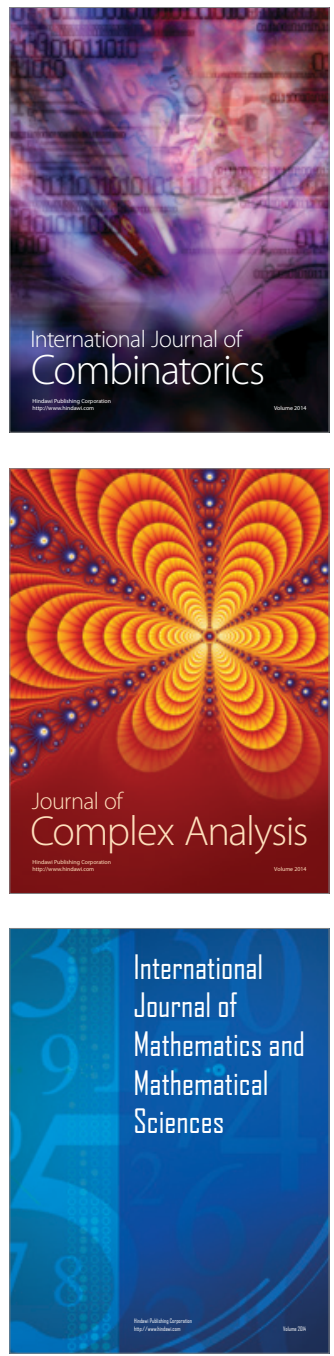
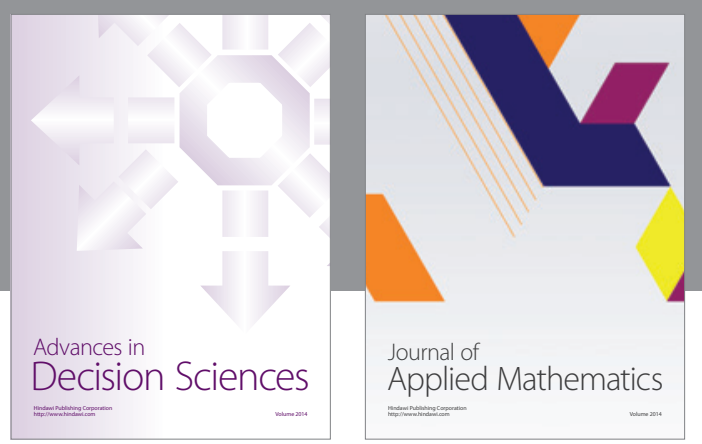

Algebra

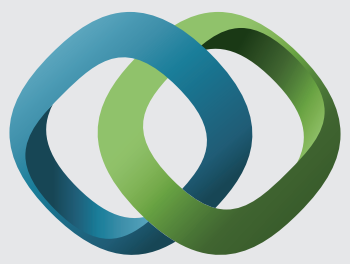

\section{Hindawi}

Submit your manuscripts at

http://www.hindawi.com
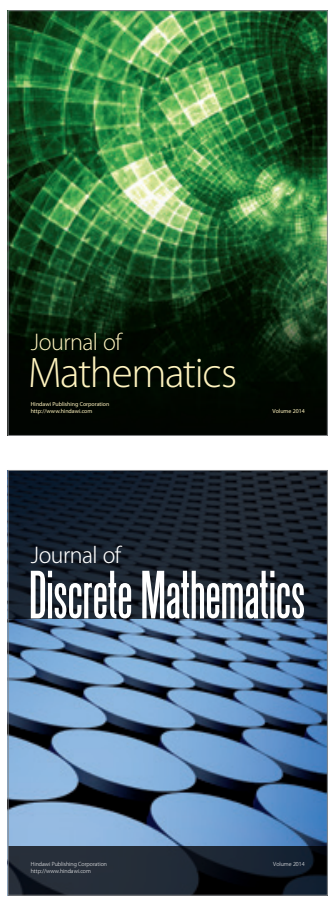

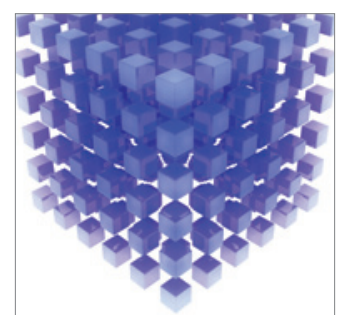

Mathematical Problems in Engineering
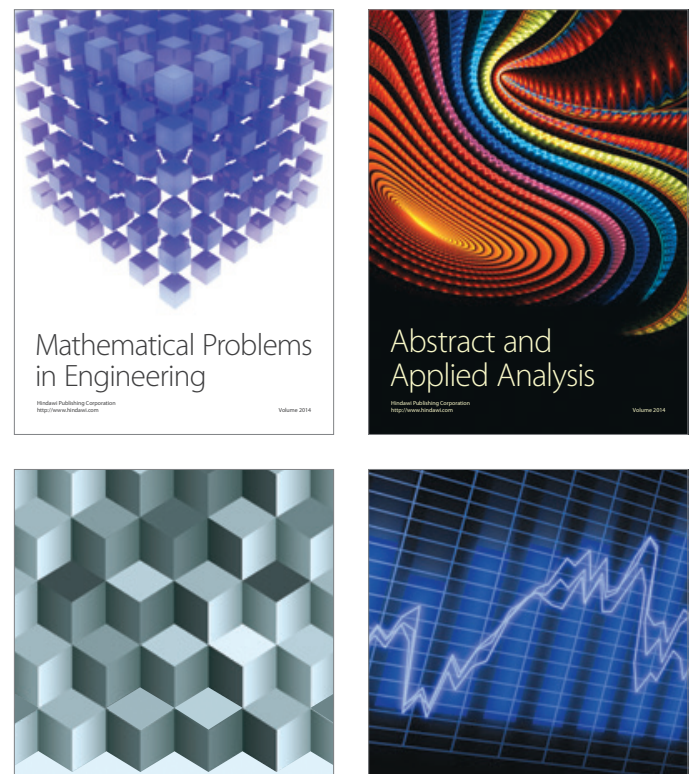

Journal of

Function Spaces

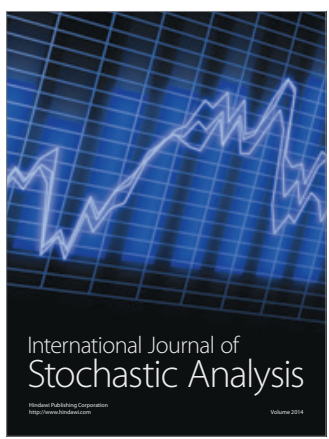

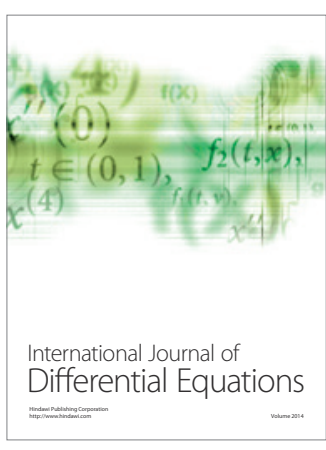
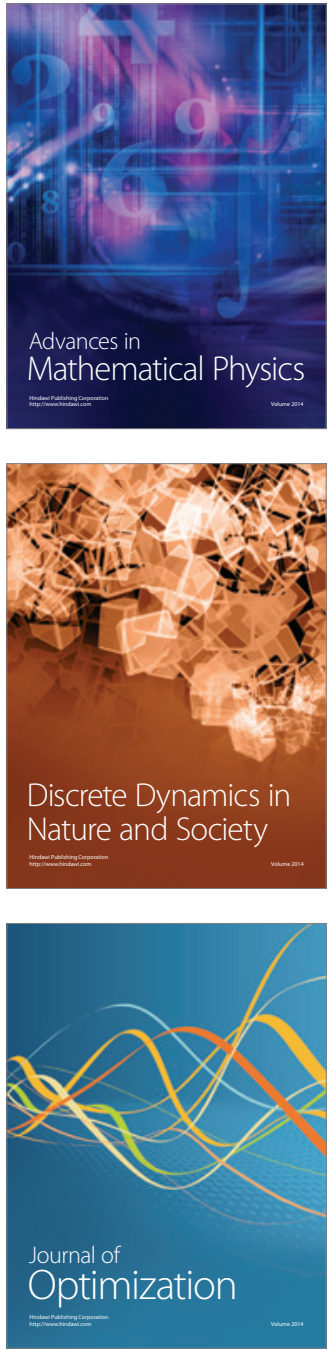